\title{
RESULTADOS DO TRANSPLANTE HEPÁTICO EM PACIENTES COM DIAGNÓSTICO PRÉ-OPERATÓRIO DE HEPATOCARCINOMA
}

\author{
Mônica Beatriz PAROLIN ${ }^{1}$, Júlio Cezar Uili COELHO ${ }^{1}$, Jorge Eduardo Fouto MATIAS ${ }^{1}$, \\ Giorgio A. P. BARETTA ${ }^{2}$, Sérgio Ossamu IOSHII $^{3}$ e Hygor NARDO 4
}

RESUMO - Racional - O hepatocarcinoma é o tumor hepático maligno mais freqüente em humanos e sua forte associação com a cirrose torna a abordagem terapêutica ainda um desafio. O transplante hepático é o tratamento de escolha para os pacientes cirróticos com hepatocarcinoma irressecável em estágio precoce. Objetivo - Avaliar a evolução pós-transplante de 15 pacientes cirróticos com diagnóstico pré-operatório de hepatocarcinoma precoce irressecável que preenchiam os Critérios de Milão e foram submetidos a transplante hepático entre setembro de 1991 e dezembro de 2003 no Hospital de Clínicas da Universidade Federal do Paraná, Curitiba, PR. Métodos - Foram analisados retrospectivamente os dados clínicos dos 15 receptores de transplante hepático tendo como parâmetros principais a sobrevida do paciente e a ocorrência de recidiva tumoral após o transplante. Resultados - A idade média dos pacientes foi 49,2 $\pm 14,3$ anos, sendo a hepatite crônica $\mathrm{C}$ a causa implicada em $60 \%$ dos casos. Em $80 \%$ dos pacientes realizou-se terapia adjuvante pré-transplante (quimioembolização ou alcoolização). Na análise dos explantes hepáticos constatou-se que 5 dos 12 pacientes (38,5\%) submetidos a terapia adjuvante apresentavam necrose completa do tumor, enquanto os demais apresentavam alguma extensão de tecido tumoral viável. Em apenas 4 dos 15 explantes (26,6\%) constatou-se invasão microvascular. A mediana do seguimento pós-transplante foi de 33 meses (8-71 meses), tendo ocorrido apenas um óbito precoce, não relacionado à recidiva tumoral (sepse biliar). A sobrevida pós-transplante livre de recurrência em 1 e 3 anos no grupo foi de $93 \%$. Conclusões - O transplante hepático é uma excelente alternativa de tratamento em pacientes cirróticos com hepatocarcinoma irressecável em fase precoce, alcançando elevados índices de sobrevida livre de recurrência tumoral.

DESCRITORES - Transplante de fígado. Carcinoma hepatocelular. Neoplasias hepáticas. Cirrose hepática.

\section{INTRODUÇÃo}

O hepatocarcinoma (HCC) é uma complicação comum nos doentes com cirrose hepática, com risco de instalação de $1 \%$ a $3 \%$ ao ano nessa população ${ }^{(4)}$. A ressecção cirúrgica e o transplante hepático (TH) são as opções de tratamento curativo nos pacientes cirróticos com tumor restrito ao fígado em estágio precoce ${ }^{(5,12,17)}$.

O TH é considerado uma intervenção terapêutica altamente efetiva em pacientes com cirrose complicada pela presença de $\mathrm{HCC}$, visto que remove o tumor e o fígado cirrótico, território com potencial neoplásico. Os candidatos mais adequados para o TH são aqueles com tumor único com diâmetro não superior a $5 \mathrm{~cm}$ ou até três lesões com diâmetro individual de até $3 \mathrm{~cm}$, sem evidência de invasão vascular macroscópica ou metástase extra-hepática ${ }^{(17)}$. Tais critérios, conhecidos como Critérios de Milão, têm sido atualmente adotados em considerável número de centros transplantadores e associam-se a índices de sobrevida em 5 anos superiores a $70 \%$ e recurrência tumoral inferior a $15 \%(13,17)$.

O objetivo do presente estudo foi avaliar a evolução pós-transplante dos pacientes cirróticos com diagnóstico pré-operatório de HCC que preenchiam os Critérios de Milão e que foram submetidos a transplante de fígado no Serviço de Transplante Hepático do Hospital de Clínicas da Universidade Federal do Paraná (STH-HC/UFPR), particularmente no que diz respeito à sobrevida pós-transplante e ocorrência de recidiva tumoral.

Trabalho realizado nos Serviços de Transplante Hepático e de Anatomia Patológica do Hospital de Clínicas da Universidade Federal do Paraná

${ }^{1}$ Serviço de Transplante Hepático; ${ }^{2}$ Serviço de Cirurgia do Aparelho Digestivo; ${ }^{3}$ Serviço de Anatomia Patológica do Hospital de Clínicas da Universidade Federal do Paraná - UFPR; ${ }^{4}$ Faculdade de Medicina da UFPR, Curitiba, PR.

Endereço para correspondência: Dra. Mônica Beatriz Parolin - Rua Lamenha Lins, 2280 - 80220-080 - Curitiba, PR. E-mail: mbparolin@hotmail.com 


\section{PACIENTES E MÉTODOS}

\section{Pacientes}

A análise retrospectiva do banco de dados dos 282 pacientes submetidos a transplante hepático no STH/HC-UFPR no período entre setembro de 1991 e dezembro de 2003, identificou 15 indivíduos $(5,23 \%)$ com diagnóstico pré-operatório de HCC, os quais foram selecionados para o presente estudo. O diagnóstico pré-operatório do HCC foi estabelecido mediante critérios semelhantes aos propostos pela Conferência da Associação Européia para Estudos das Doenças do Fígado (EASL) ${ }^{(6)}$ e baseou-se na presença de lesão tumoral intra-hepática hipervascularizada na fase arterial da tomografia computadorizada (TC) ou ressonância magnética (RM), acompanhada de taxas elevadas de alfa-fetoproteína ou característica hipervascular à arteriografia hepática. Apenas 1 dos 15 pacientes foi submetido a biopsia hepática com finalidade diagnóstica. Como parte da avaliação pré-transplante dos candidatos com HCC, todos foram submetidos a exame de ecodoppler de vasos hepáticos, TC de tórax e de crânio e cintilografia óssea para exclusão de invasão vascular e doença extra-hepática.

Conforme protocolo do Serviço, o critério de elegibilidade para o transplante adotado nos doentes com HCC foi o proposto por MAZZAFERRO et al. ${ }^{(17)}$. A única exceção consistiu num paciente que apresentava à RM tumor único de $5,7 \mathrm{~cm}$ de diâmetro restrito ao fígado, o qual foi submetido a uma sessão de quimioembolização antes da realização de transplante hepático intervivos. $\mathrm{O}$ exame do explante hepático demonstrou necrose completa do tumor e ausência de invasão vascular tumoral.

Durante o período em que os pacientes permaneceram em lista aguardando o transplante, realizaram periodicamente exames de imagem para monitorar progressão do tumor e surgimento de metástases. Quando a perspectiva de permanência em lista de espera era longa ou não havia possibilidade de transplante intervivos, indicou-se a realização de tratamento adjuvante (preferencialmente quimioembolização seletiva do ramo da artéria hepática nutridora do tumor). Esta era oferecida aos pacientes com reserva funcional razoável (Child A ou B) e com veia porta permeável, porém na impossibilidade de ser realizada(problemas técnicos e/ou disfunção hepática grave), os pacientes eram submetidos a alcoolização percutânea das lesões. A terapia adjuvante era realizada periodicamente, na persistência de lesão de padrão hipervascular nos métodos de imagem (RM ou TC).

As principais características demográficas e clínicas pré-operatórias dos 15 pacientes selecionados para o estudo estão dispostas na Tabela 1 . Todos os pacientes eram do sexo masculino e a principal causa da doença hepática subjacente foi a hepatite $\mathrm{C}$, respondendo por 9 dos $15(60 \%)$ casos. A mediana do tempo decorrido entre o diagnóstico do tumor e a realização do transplante foi de 4 meses, variando de 1,5 a 19 meses. Sete dos 15 pacientes $(46,66 \%)$ apresentavam boa reserva funcional (Child A) e a cirurgia foi motivada pela presença de tumor irressecável. A concentração sérica de alfa-fetoproteína obtida na época de inclusão em lista encontrava-se em taxas superiores a $100 \mathrm{ng} / \mathrm{mL}$ em 8 dos 15 pacientes $(53,33 \%)$.
TABELA 1 - Características gerais de 15 pacientes com HCC submetidos a transplante hepático

\begin{tabular}{lc}
\hline Variáveis & $\mathbf{n}(\%)$ \\
\hline Idade média (anos) & $49,2 \pm 14,3$ \\
Etiologia da doença hepática & \\
$\quad$ Hepatite C & $9(60 \%)$ \\
Hepatite B & $3(20 \%)$ \\
$\quad$ Álcool & $1(6,66 \%)$ \\
$\quad$ Criptogênica & $1(6,66 \%)$ \\
Cirrose biliar primária & $1(6,66 \%)$ \\
Child-Pugh & \\
A / B / C & $7 / 7 / 1$ \\
Indicação do transplante & \\
Cirrose descompensada + HCC & $8(53,33 \%)$ \\
HCC não ressecável & $7(46,66 \%)$ \\
Número de lesões & \\
Unicêntrico / multicêntrico & $11 / 4$ \\
Taxa de alfa-fetoproteína (ng/mL) & \\
$<$ 20 & \\
20-100 & $4(26,66 \%)$ \\
$>100$ & $3(20 \%)$ \\
Terapia adjuvante & $8(53,33 \%)$ \\
Quimioembolização & $12(80 \%)$ \\
Alcoolização & 10 \\
Quimioembolização + alcoolização & 1 \\
&
\end{tabular}

De acordo com os exames de imagem realizados no pré-operatório, 11 pacientes $(73,33 \%)$ apresentavam tumor unicêntrico. Doze $(80 \%)$ receberam algum tipo de terapia para o $\mathrm{HCC}$ na fase pré-transplante. Dez foram submetidos exclusivamente a quimioembolização com mitomicina $\mathrm{C}$ e lipoidol, variando de uma a quatro sessões. Um paciente foi submetido a uma sessão de quimioembolização e uma de alcoolização, e um paciente foi submetido a uma única sessão de alcoolização. Nenhum paciente apresentou descompensação da doença hepática após receber terapia adjuvante.

\section{Avaliação histológica}

Os explantes hepáticos foram analisados por patologista experiente para a avaliação das características histopatológicas das lesões identificadas tais como número, tamanho, presença de tumor viável nos pacientes submetidos a tratamento neoadjuvante pré-transplante, presença de invasão macro ou microvascular, grau histológico do tumor de acordo com a classificação de EDMONDSON e STEINER ${ }^{(8)}$ e presença de metástases em linfonodos.

\section{Seguimento pós-transplante e rastreamento para recurrência tumoral}

Foram revisados prontuários para verificar o tipo de esquema de imunossupressão adotado, tempo de hospitalização póstransplante, ocorrência de recidiva tumoral e causa de óbito. O protocolo adotado no Serviço para rastrear recidiva tumoral após TH consta da realização de alfa-fetoproteína, TC de tórax, crânio e abdome e cintilografia óssea a cada 4 meses no primeiro ano, a cada 6 meses no segundo ano e a cada 12 meses, a partir de então. 
Análise estatística

Os resultados foram expressos como média ( \pm desvio padrão) ou mediana (variação) para as variáveis contínuas e percentagens para as variáveis binomiais. O teste de $t$ Student foi usado para analisar a significância de diferenças nas médias e o teste não-paramétrico de Wilcoxon rank sum foi usado para comparar valores de medianas. As variáveis categóricas foram comparadas através do teste exato de Fischer. Em todos os testes considerou-se o nível de significância de $5 \%(P<0,05)$.

\section{RESULTADOS}

Dos 15 pacientes avaliados, $10(66,66 \%)$ receberam enxerto cadavérico e $5(33,33 \%)$ enxerto de doador vivo. O esquema de imunossupressão pós-transplante adotado em $80 \%$ dos pacientes (12/15) foi o tacrolimus em associação com doses baixas de prednisona, enquanto nos demais empregou-se ciclosporina como inibidor de calcineurina. A mediana do tempo de hospitalização pós-transplante foi de 12 dias, variando de 8 a 38 dias.

A análise histopatológica dos explantes hepáticos revelou a presença de tumor viável em 10 dos 15 pacientes transplantados. Entre os 12 pacientes submetidos a terapia adjuvante pré-transplante, $5(41,66 \%)$ apresentavam necrose total do tumor, enquanto nos 7 demais observaram-se graus variáveis de tecido tumoral viável. As principais características dos tumores encontrados nos explantes estão dispostas na Tabela 2. Não houve invasão linfática na amostra estudada.

TABELA 2 - Achados histopatológicos nos explantes de 15 pacientes cirróticos com HCC

\begin{tabular}{lc}
\hline Variáveis & $\mathrm{n}(\%)$ \\
\hline Tumor viável no explante (n, \%) & $10 / 15(66,66)$ \\
Distribuição das lesões viáveis no explante & \\
$\quad$ Solitária & $6 / 10(60 \%)$ \\
$\quad$ Múltipla & $4 / 10(40 \%)$ \\
Diâmetro médio das lesões viáveis (cm, variação) & $3,18(2-5)$ \\
Grau Histológico (n, \%) & \\
1 - Bem diferenciado & $2(20 \%)$ \\
2 - Moderadamente diferenciado & $6(60 \%)$ \\
3 - Pouco diferenciado & $2(20 \%)$ \\
Invasão microvascular (n, \%) & $4(40 \%)$ \\
\hline
\end{tabular}

Não houve diferença estatisticamente significante entre o número de lesões e a presença de invasão vascular $(P=0,23)$. Da mesma forma, o tamanho da lesão baseada em exames de imagem e no exame do explante não foi estatisticamente diferente de acordo com a presença ou não de invasão vascular $(P=0,21)$. Não houve diferença da taxas de alfa-fetoproteína de acordo com o número de lesões $(P=0,88)$, sendo observado coeficiente de correlação positivo $(P=0,51)$ entre as taxas de alfa-fetoproteína e o tamanho das lesões.

Apenas um óbito foi registrado 2 meses após o transplante, devido à sepse de origem biliar. A mediana do período de acompanhamento pós-transplante nos demais 14 pacientes foi de 33 meses ( 8 a 71 meses) e a sobrevida livre de recurrência em 1 e 3 anos após o transplante foi de 93,3\%. Nenhum paciente necessitou de re-transplante e nenhum caso de recidiva tumoral foi detectado até o momento.

\section{DISCUSSÃO}

Os resultados do presente estudo confirmam as observações prévias de que o transplante é ótima opção terapêutica para indivíduos cirróticos com hepatocarcinoma irressecável em fase precoce. Os 14 pacientes com seguimento pós-transplante superior a 6 meses apresentaram sobrevida livre de recidiva tumoral de $93 \%$ em 3 anos. A seleção apropriada dos candidatos a transplante é de fundamental importância para se otimizar os resultados e assegurar o melhor aproveitamento possível dos órgãos doados. Embora a amostra desta série seja reduzida, os índices de sobrevida livre de recurrência tumoral encontrados, assemelham-se aos relatados em séries maiores MAZZAFERRO et al. ${ }^{(17)}$ demonstraram sobrevida livre de recurrência de $92 \%$ em 4 anos em pacientes com tumores em fase T2, comparado com $59 \%$ em tumores mais avançados. No estudo de FIGUERAS et al. ${ }^{(9)}$ observou-se que a presença de tumor $>5 \mathrm{~cm}$ associou-se à acentuada redução da sobrevida 1 ano após transplante $(25 \%$ comparada a $88 \%$ nos doentes com tumores $<5 \mathrm{~cm}$ ). Também BISMUTH et al..$^{(3)}$ documentaram o impacto negativo de tumores maiores na sobrevida pós-transplante (sobrevida 5 anos de $42 \% \mathrm{em}$ tumores $>5 \mathrm{~cm}$ vs. $66 \%$ em tumores $<3 \mathrm{~cm}$ ). Mais recentemente, SHETTY et al. ${ }^{(23)}$ analisando 109 pacientes com HCC submetidos a transplante na Universidade da Pensilvânia, encontraram sobrevida livre de recurrência em 1 e 3 anos de $89 \%$ e $75 \%$, respectivamente. $\mathrm{O}$ tamanho do tumor no explante apresentou forte correlação com invasão vascular e multifocalidade, sendo que tumores classificados como pelo menos T3 (um nódulo $>5$ $\mathrm{cm}$ : dois ou três nódulos, sendo um $>3 \mathrm{~cm}$ ) apresentaram maior risco de recurrência.

Tamanho do tumor, invasão vascular, grau histológico pouco diferenciado e, em alguns estudos, doença bilobar, são identificados como importantes indicadores de recurrência ${ }^{(18)}$. Em pacientes com tumor precoce, a invasão vascular aparece como fator de risco para recurrência. Este achado é responsável por alguns dos casos de recurrência que ocorreram com pacientes transplantados dentro dos Critérios de Milão (4\% a 17\% dos

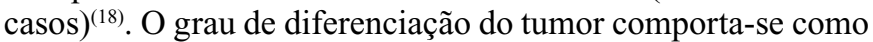
marcador indireto do potencial de invasão vascular. Habitualmente a graduação histológica aumenta à medida que o tumor cresce, ou seja, lesões menores demonstram histologia bem diferenciada e tumores maiores tipicamente apresentam áreas moderadamente ou pouco diferenciadas ${ }^{(24)}$. Ocasionalmente, tumores pequenos podem se apresentar pouco diferenciados. $\mathrm{Na}$ experiência do Hospital Mount Sinai, observou-se recurrência do tumor póstransplante em 4 de 20 pacientes com tumor $\leq 3 \mathrm{~cm}$, todos pouco diferenciados e com invasão vascular microscópica, apesar do pequeno tamanho das lesões ${ }^{(21)}$. No presente estudo nenhum caso de recurrência tumoral foi documentado durante o período de seguimento pós-transplante (mediana de 33 meses, variando de 8 a 71 meses). Quatro pacientes apresentaram invasão microvascular 
no exame do explante, achado que não se correlacionou de modo estatisticamente significante com o número ou o tamanho das lesões. Não se observou influência da presença de invasão microvascular na sobrevida livre de recidiva durante a mediana de tempo de seguimento de 33 meses. Dos quatro pacientes com invasão microvascular, dois apresentavam tumor moderadamente diferenciado e dois pouco diferenciado. Em que pesem os dados existentes na literatura a respeito da implicação da invasão vascular na recurrência tumoral pós-transplante, esse achado é um marcador imperfeito da agressividade tumoral e estudos sobre o perfil genético dos HCC poderão fornecer subsídios para estratificação mais acurada dos pacientes com HCC, de acordo com o risco de recurrência do tumor ${ }^{(14,18)}$.

Embora a recurrência do HCC ocorra, na maioria das vezes, nos 2 primeiros anos após o TH, tal complicação já foi documentada após intervalos maiores. ROAYAIE et al. ${ }^{(19)}$ ao analisarem 311 pacientes com HCC submetidos a transplante, encontraram taxa de recurrência de $18 \%$. A mediana da época em que foi diagnosticada a recurrência foi 12 meses pós-TH, variando de 1,5 a 60 meses. Cerca de $10 \%$ das recurrências ocorreram após 4 anos do transplante. Os fatores associados a maior chance de recurrência, foram tumor $\geq 5 \mathrm{~cm}$ e grau histológico pouco diferenciado, achados que também se correlacionaram com recurrência precoce e menor sobrevida pós-transplante.

Além das características tumorais (por exemplo: tamanho, invasão vascular, grau de diferenciação histológica), um aspecto que tem sido investigado quando à possibilidade de influenciar a taxa de recurrência, é o emprego dos imunossupressores. No presente estudo, a maioria dos pacientes estava sob imunossupressão com tacrolimus. Dados preliminares advindos de recente publicação de VIVARELLI et al. ${ }^{(25)}$, sugerem que a intensidade de exposição à ciclosporina seria fator de risco para recurrência tumoral. Embora a amostra desse estudo seja reduzida, tais dados estimulam novos estudos nessa direção. Sabe-se que os inibidores de calcineurina fazem muito mais que prevenir episódios de rejeição do enxerto. Tais medicamentos favorecem o crescimento tumoral através de múltiplos mecanismos. Por outro lado, o imunossupressor sirolimus tem se mostrado capaz de inibir ampla variedade de tumores, através de mecanismos que incluem interferência na angiogênese tumoral e ativação da apoptose, como demonstrados em estudos clínicos e experimentais ${ }^{(22)}$. Baseados nessas informações alguns centros de transplante estão empregando o sirolimus com ou sem inibidor da calcineurina, como imunossupressão primária pós-transplante em pacientes com $\mathrm{HCC}^{(11,22)}$.

Conforme mencionado anteriormente, 1 dos 15 pacientes incluídos no presente estudo tinha HCC com dimensão acima dos critérios de Milão (nódulo único de $5,7 \mathrm{~cm}$ ), o qual foi tratado com quimioembolização algumas semanas antes da realização do transplante intervivos. $\mathrm{O}$ exame do explante constatou a necrose total do tumor, não havendo evidência de invasão vascular ou de linfonodos. Nos últimos anos a comunidade transplantadora tem debatido calorosamente sobre a viabilidade de se ampliar, ainda que modestamente, os critérios de indicação para transplante nos doentes com HCC, desde que se assegurem resultados satisfatórios. Um dos estudos que suscitou tal discussão foi publicado pelo grupo da Universidade da Califórnia em São
Francisco (UCSF), no qual a presença no explante de lesão única $\leq 6,5 \mathrm{~cm}$ de diâmetro ou até três lesões $\leq 4,5$ e diâmetro total das lesões $\leq 8 \mathrm{~cm}$, não afetou de modo adverso a sobrevida ${ }^{(26)}$. No referido estudo, os 60 pacientes cujos tumores encontrados no explante apresentavam os Critérios da UCSF, alcançaram sobrevida em 1 e 5 anos de $90 \%$ e $75 \%$, respectivamente, comparados com sobrevida de 50\% em 1 ano nos 10 pacientes cujos tumores ultrapassaram os referidos critérios. Embora à primeira vista tais resultados sejam animadores, um dos pontos mais controversos e criticados no estudo do grupo da UCSF é o fato que tais critérios derivam da análise do explante, ao invés de se basearem em dados prospectivos obtidos de exames de imagem realizados no pré-transplante, à semelhança dos critérios de Milão. Atualmente a TC helicoidal e a RM são considerados os melhores métodos não-invasivos para o diagnóstico de HCC, com precisão estimada de $80 \%^{(18)}$. Recentemente demonstrouse que a angiorressonância é significativamente melhor que a TC helicoidal na detecção de nódulos de HCC (76\% vs. $61 \%)$, particularmente quando se consideram nódulos adicionais entre 10 a $20 \mathrm{~mm}$ de diâmetro $(84 \% \text { vs. } 41 \%)^{(7)}$ Portanto, mesmo empregando-se as melhores técnicas e equipamentos, os exames de imagem ainda podem subestimar em algum grau o estádio do HCC, particularmente na falha de detectar pequenos nódulos adicionais $<1 \mathrm{~cm}$ ou invasão vascular microscópica. Tal limitação dos métodos de imagem ainda é um dos pontos de preocupação para aqueles que defendem a expansão dos critérios atualmente adotados, pois nenhuma técnica de imagem mostrou a mesma precisão diagnóstica que o exame anatomopatológico ${ }^{(18)}$.

OTH é o único tratamento que assegura a completa remoção de todos os focos hepáticos de tumor, bem como o tecido sob risco de recurrência tumoral, resultando em índices de sobrevida livre de recidiva tumoral significativamente superiores aos obtidos pela ressecção cirúrgica e indistinguíveis dos resultados do TH em cirróticos sem malignidade hepática. A escassez de órgãos é fator limitante quando se indica o TH em doentes com HCC, pois com o crescente aumento no número de pacientes em lista, o tempo decorrido até a obtenção do órgão é, na maioria das vezes, superior a 3 anos em nosso meio. Como o tempo estimado para que o HCC dobre de tamanho varia entre 4 a 6 meses, o tumor pode progredir significativamente durante o tempo de espera em lista, aumentando o risco de invasão microvascular, metástases ocultas e recurrência pós-transplante ${ }^{(1)}$. Para superar esses obstáculos, rotineiramente é oferecido aos doentes com HCC a opção do TH intervivos, utilizando o lobo direito do doador para adultos e o lobo esquerdo para pacientes pediátricos. No presente estudo, 5 dos 15 pacientes foram submetidos a transplante hepático intervivos, sendo que 1 deles evoluiu para óbito 2 meses após, em virtude de sepse biliar.

Como a legislação brasileira vigente na época em que os pacientes foram transplantados não priorizava a distribuição de órgãos aos pacientes com HCC, nos casos em que o TH intervivos não foi possível, indicou-se terapia neo-adjuvante para controle do crescimento tumoral (preferencialmente a quimioembolização), desde que o paciente não apresentasse contra-indicações para tal. No presente estudo, o tempo decorrido entre o diagnóstico de $\mathrm{HCC}$ e a realização do $\mathrm{TH}$ nos 15 pacientes 
foi relativamente curto (mediana de 4 meses). Em 12 deles empregou-se algum tipo de terapia neo-adjuvante, na maioria das vezes quimioembolização seletiva do tumor. No STH-HC/ UFPR tem-se adotado como protocolo, sempre que possível, a realização periódica de quimioembolização seletiva para controle do crescimento tumoral nos candidatos em lista. Tal prática restringe-se aos pacientes com boa reserva funcional hepática, pelo risco elevado de grave descompensação da hepatopatia em pacientes Child C. Em 2004, pesquisadores da Clínica Mayo, Rochester, EUA, publicaram a experiência do grupo com protocolo de quimioembolização periódica em 54 portadores de HCC em lista para TH naquela instituição ${ }^{(16)}$. Os pacientes eram submetidos a sessão de quimioembolização a cada 3 meses, até a realização do transplante (dois pacientes foram classificados como tendo HCC T1, 45 T2, e 6 T3, no momento de inclusão em lista). A mediana de tempo de espera em lista foi de 211 dias e a taxa de remoção da mesma, devido à progressão tumoral após 6 meses da inclusão, foi de $15 \%$. Como a quimioembolização pode retardar o crescimento tumoral e conseqüentemente a invasão vascular, bem como prolongar a sobrevida, este método tem sido amplamente utilizado em muitos centros transplantadores enquanto se aguarda a realização do transplante ${ }^{(20)}$.

Mais recentemente, a ablação por radiofreqüência via percutânea vem ganhando espaço como boa alternativa para controle do HCC nos pacientes em lista para transplante. Essa técnica, apropriada para pacientes com reserva funcional hepática adequada (Child A ou B) e nódulos de localização segura e acessível, apresenta alta eficácia na destruição do tumor. No exame dos explantes de 52 pacientes com 87 nódulos tumorais submetidos a essa técnica constatou-se necrose coagulativa completa de 74 dos 87 nódulos $(85 \%)^{(15)}$. Nesse estudo o emprego da ablação por radiofreqüência resultou na exclusão de apenas 3 dos $52(5,8 \%)$ pacientes com HCC, acompanhados por média de 12,7 meses em lista. Os autores encontraram sobrevida pós-transplante de 1 e 3 anos de, respectivamente, $85 \%$ e $76 \%$, não sendo registrado nenhum caso de recurrência. A radiofreqüência ainda tem sido pouco indicada no Brasil pelo elevado custo do procedimento e cobertura limitada pelos convênios médicos e pelo SUS

Apesar de $60 \%$ dos pacientes na presente série apresentarem com causa subjacente da doença hepática a infecção pelo VHC, não houve evidências de que esta infecção tenha influenciado negativamente a sobrevida pós-TH durante o período analisado. Entretanto, o período disponível de seguimento pós-transplante ainda é limitado para que se possa avaliar mais profundamente o impacto da recidiva viral na sobrevida desses pacientes. Sabe-se que pacientes portadores de hepatite $\mathrm{C}$ submetidos a transplante hepático podem apresentar sobrevida reduzida em relação a outras etiologias, devido à recurrência viral no enxerto com progressão até cirrose ${ }^{(2,10)}$.

As limitações do presente estudo, de natureza retrospectiva, incluem amostra de tamanho reduzido e inexistência de recidiva tumoral ou óbito relacionado ao HCC no período de seguimento, o que prejudicou análises estatísticas correlacionando prováveis fatores de risco para recidiva tumoral e prognóstico de sobrevida nessa população.

\section{CONCLUSÃO}

$\mathrm{O} \mathrm{TH}$, quando realizado em pacientes cirróticos com HCC em fase precoce, de acordo com os Critérios de Milão, resulta em excelentes índices de sobrevida livre de recurrência tumoral. Esses resultados enfatizam a importância da seleção apropriada dos candidatos com tumor.

Parolin MB, Coelho JCU, Matias JEF, Baretta GAP, Ioshii SO, Nardo H. Results of liver transplantation in patients with preoperative diagnosis of hepatocellular carcinoma. Arq Gastroenterol. 2006;43(4):259-64.

ABSTRACT - Background - Hepatocellular carcinoma is the most frequent malignant hepatic tumor in humans, and its association with cirrhosis makes the therapeutic approach still a challenge. Liver transplantation is the treatment of choice for cirrhotic patients with unresectable early hepatocellular carcinoma. Aim - To evaluate the post-transplant outcome of a cohort of 15 cirrhotic patients with preoperative diagnosis of unresectable early hepatocellular carcinoma according the Milan criteria who underwent liver transplantation between September 1991 and December 2003. Methods - We retrospectively reviewed the clinical data from 15 liver transplant recipients and the explanted livers were assessed for the efficacy of preoperative therapy. Patient survival and tumor recurrence were evaluated as primary outcome measures. Results - The mean age of the patients was $49.2 \pm 14.3$ years and hepatitis $C$ was the etiology of the underlying liver disease in $60 \%$. Preoperative therapy, either chemoembolization or percutaneous ethanol injection, was performed in $12(86 \%)$ patients. Complete necrosis of all tumoral lesions were observed in 5 of 12 patients (44,66\%); all others had variable amounts of viable tumor in the explanted liver. Only 4 of the 15 (26.6\%) explanted livers had microscopic vascular invasion. The median post-transplant follow-up was 33 months (range: $8-71$ months) and no tumor recurrence was detected during this period. The only death was an early event not related to the tumor. The recurrence-free survival rates at 1 and 3 years were $93 \%$. Conclusion - Liver transplantation has emerged as a good alternative for cirrhotic patients with early hepatocellular carcinoma not amenable to curative resection, offering excellent recurrence-free survival rates.

HEADINGS - Liver transplantation. Carcinoma, hepatocellular. Liver neoplasms. Liver cirrhosis. 


\section{REFERÊNCIAS BIBLIOGRÁFICAS}

1. Barbara L, Benzi G, Gaiani S, Fusconi F, Zironi G, Siringo S, Rigamonti A, Barbara C, Grigioni W, Mazzioti A. Natural history of small untreated hepatocellular carcinoma in cirrhosis: a multivariate analysis of prognostic factors of tumor growth rate and patients survival. Hepatology. 1992;16:132-7.

2. Berenguer M, Prieto M, Rayon JM, Mora J, Pastor M, Ortiz V, Carrasca D, San Juan F, Burguerro MD, Mir J, Berenguer J. Natural history of clinically compensated HCVrelated graft cirrhosis following liver transplantation. Hepatology. 2000;32:852-8

3. Bismuth H, Chiche L, Adam R, Castaing D, Diamond T, Denison A. Liver resection versus transplantation for hepatocellular carcinoma in cirrhotic patients. Ann Surg. 1993;218:145-51.

4. Bosch FX, Ribes J, Borras J. Epidemiology of primary liver cancer. Semin Liver Dis. 1999;19:271-85

5. Bruix J, Castells A, Bosch J, Feu F, Fuster J, Garcia-Pagan JC, Visa J, Bruix C, Rodes J. Surgical resection of hepatocellular carcinoma in cirrhotic patients: prognostic value of preoperative portal pressure. Gastroenterology. 1996;111:1018-22.

6. Bruix J, Sherman M, Llovet JM, Beaugrand M, Lencioni R, Burroughs AK, Chrisyensen E, Pagliaro L, Colombo M, Rodés J. Clinical management of hepatocellular carcinoma: conclusions of the Barcelona-2000 EASL Conference. J Hepatol. 2001;35:421-30.

7. Burrel M, Llovet JM, Ayuso C, Iglesias C, Sala M, Miguel R, Caralt T, Ayuso JR, Sole M, Sanchez M, Bru C, Bruix J, Barcelona Clinic Liver Cancer Group. MRI angiography is superior to helical CT for detection of HCC prior to liver transplantation: an explant correlation. Hepatology. 2003;38:1134-42.

8. Edmondson HA, Steiner PE. Primary carcinoma of the liver. A study of 100 cases among 48,900 neoplasms. Cancer. 1954;7:462-503.

9. Figueras J, Jaurrieta E, Valls C, Benasco C, Rafecas A, Xiol X, Fabregat J, Casanovas T, Torras J, Baliellas C, Ibanez L, Moreno P, Casais L. Survival after liver transplantation in cirrhotic patients with and without hepatocellular carcinoma: a comparative study. Hepatology. 1997;25:1485-9.

10. Ganes $\mathrm{E}$. The natural history and outcome of liver transplantation in hepatitis $\mathrm{C}$ virusinfected recipients. Liver Transpl. 2003;9:s28-s34.

11. Kneteman NM, Oberholzer J, Al Saghier M, Meeberg GA, Blitz M, Ma MM, Wong WW, Gutfreund K, Mason AL, Jewell LD, Shapiro AM, Bain VG, Bigam DL. Sirolimusbased immunossupression for liver transplantation in the presence of extended criteria for hepatocellular carcinoma. Liver Transpl. 2004;10:1301-11.

12. Llovet JM, Fuster J, Bruix J. Intention-to-treat analysis of surgical treatment for early hepatocellular carcinoma: resection versus transplantation. Hepatology. 1999:39:1434-40.

13. Llovet JM, Fuster J, Bruix J. The Barcelona approach: diagnosis, staging, and treatment of hepatocellular carcinoma. Liver Transpl. 2004;10:s115-s20.
14. Llovet JM, Wurmbach E. Gene expression profiles in hepatocellular carcinoma: not yet there. J Hepatol. 2004;41:336-9.

15. Lu DSK, Yu NC, Raman SS, Lassman C, Tong MJ, Britten C, Durazo F, Saab S, Han S, Finn R, Hiatt JR, Busuttil RW. Percutaneus radiofrequency ablation of hepatocellular carcinoma as a bridge to liver transplantation. Hepatology. 2005;41:1130-7.

16. Maddala Y, Stadheim L, Andrews JC, Burgart LJ, Rosen CB, Kremers WK, Gores $\mathrm{G}$. Drop-out rates patients with hepatocellular cancer listed for liver transplantation: outcome with chemoembolization. Liver Transpl. 2004;10:449-55.

17. Mazzaferro V, Regalia E, Doci R, Andreola S, Pulvirenti A, Bozzeti F, Montolto F, Annaturoni M, Moobito A, Gennari L. Liver transplantation for the treatment of small hepatocellular carcinomas in patients with cirrhosis. N Engl J Med. 1996;334:693-9.

18. Roayaie S, Llovet JM. Liver transplantation for hepatocellular carcinoma: is expansion of criteria justified? Clin Liver Dis. 2005;9:315-28.

19. Roayaie S, Schwartz JD, Sung MW, Emre SH, Miller CM, Gondolesi GE, Krieger NR, Schwartz ME. Recurrence of hepatocellular carcinoma after liver transplant: patterns and prognosis. Liver Transpl. 2004;10:534-40.

20. Sala M, Varela M, Bruix J. Selection of candidates with HCC for transplantation in the MELD era. Liver Transpl. 2004;10:s4-s9.

21. Schwartz M. Liver transplantation in patients with hepatocellular carcinoma. Liver Transpl. 2004;109:s81-s5.

22. Schwartz M, Konstadoulakis M, Roayaie S. Recurrence of hepatocellular carcinoma after liver transplantation: is immunosuppression a factor? Liver Transpl. 2005;11:494-6.

23. Shetty K, Timmins K, Brensinger C, Furth EE, Rattan S, Sun W, Rosen M, Soulen M, Shaked A, Reddy KR, Olthoff KM. Liver transplantation for hepatocellular carcinoma validation of present selection criteria in predicting outcome. Liver Transpl. 2004;10:911-8.

24. Sugihara S, Nakashima O, Kojiro M, Majima Y, Tanaka M, Tanikawa K. The morphologic transition in hepatocellular carcinoma. A comparison of the individual histologic features disclosed by ultrasound-guided fine-needle biopsy with those of autopsy. Cancer. 1992; 70:1488-92.

25. Vivarelli M, Cucchetti A, Piscaglia F, La Barba G, Bolondi L, Cavallari A, Pinna AD. Analysis of risk factors for tumor recurrence after liver transplantation for hepatocellular carcinoma: a key role of immunosuppression. Liver Transpl. 2005;11:497-503.

26. Yao FY, Ferrell L, Bass NM, Watson JJ, Bacchetti P, Venook A. Liver transplantation for hepatocellular carcinoma: expansion of tumor size limits does not adversely impact survival. Hepatology. 2001;33:1394-403. 